\title{
Atuação do profissional de enfermagem do trabalho na prevenção do câncer de próstata
}

\author{
Graziele Carolina de Almeida Marcolin*, Amanda Conrado Silva Barbosa**, Alan Rodrigues de Souza***, \\ Tânia Cristina de Lima Sabino****, Mateus Marcolin ${ }^{* * * * *}$
}

\begin{abstract}
*Terapeuta Ocupacional, APAE Congonhas e NASF da Prefeitura Municipal de Conselheiro Lafaiete, Especialista em Desenvolvimento Infantil (UFMG), Gestão Pública de Organização de Saúde (UFJF), Formação Pedagógica para Profissionais de Saúde - CEFPEPS (UFMG), Especializando em Educação Especial e Inclusiva com Ênfase em Deficiência Múltipla e Intelectual (UCAMPROMINAS), Especializando em Planejamento, Implementação e Gestão de Cursos a Distância (UFF), **Enfermeira, Especialista em Enfermagem do Trabalho pela Faculdade Redentor/RJ, Especializando em Formaçáo Pedagógica para Profissionais de Saúde - CEFPEPS(UFMG), Docente na Rede Doctum de Ensino, Leopoldina/MG, ***Enfermeiro, Especialista em Gestão Pública de Organização de Saúde (UFJF), Especializando em Formação Pedagógica para Profissionais da Saúde-CEFPEPS (UFMG), Coordenador e Enfermeiro titular da Estratégia de Saúde da Família - Sebastião de Araújo Teixeira, no Município de Cristiano Otoni, ${ }^{* * * *}$ Enfermeira triagista no Hospital Raymundo Campos, Especializando em Gestäo de Programas de Saúde da Familia e Enfermagem do Trabalho pela Universidade Candido Mendes - Instituto Prominas -

UCAMPROMINAS, Especialista em Protocolo de Manchester pelo Canal Minas Saúde - Ministério da Saúde, Mestranda em Saúde Pública pela Universidade das Américas - UNIAMERICAS, ****Bacharelando em Psicologia (UNIPAC - Barbacena)
\end{abstract}

\section{Resumo}

O câncer de próstata é uma das patologias mais frequentes e comuns na população masculina, porém a discussão sobre sua prevenção ainda é muito defasada e necessita de maior atenção no âmbito da saúde pública. Pensando nisso, o objetivo do presente estudo é avaliar como se dá a assistência do enfermeiro do trabalho na prevenção do câncer de próstata. Para tal, o presente estudo contempla uma revisão de literatura, observando, a partir da utilização de livros, teses, monografias e artigos, responder o problema norteador que orienta tal pesquisa. Assim, foram utilizadas as palavras-chave: enfermeiros, câncer de próstata, adenocarcinoma prostático, saúde do homem, de forma combinada para coleta dos estudos incluídos na pesquisa. O idioma foi limitado ao português e o ano de publicação dos artigos incluídos limitou-se aos anos de 1990 a 2014. Dessa maneira, foram encontrados 217 artigos nas bases de dados, mas após leitura na íntegra, apenas 13 foram utilizados para a compilação dos dados da presente revisão bibliográfica. Logo, como resultado observou-se que, atualmente, o enfermeiro é o profissional mais apto a praticar o cuidado, orientação e auxílio à prevenção de homens quanto ao problema do câncer de próstata. Além disso, conforme análise do estudo, o profissional de enfermagem normalmente é o responsável pela capacitaçáo da equipe e pode auxiliar na desmistificaçáo dos pré-conceitos existentes acerca do exame de toque retal em homens. Dessa maneira, o estudo observou que o enfermeiro é um profissional indispensável no cuidado e prevenção do câncer de próstata, uma vez que apresenta conhecimentos específicos que podem auxiliar no repasse de informaçōes e orientaçóes pertinentes a esta patologia.

Palavras-chave: enfermeiros, câncer de próstata, adenocarcinoma prostático, saúde do homem. 


\section{Abstract \\ Work nursing professional procedure in the prostate cancer prevention}

Prostate cancer is one of the most frequent and common pathologies in the male population, and the discussion about its prevention is still very outdated and needs more attention in the public health context. Thinking about it, the aim of this study is to evaluate how is the assistance of the work nursing professional in the prevention of prostate cancer. For such, this study includes a literature review from books, theses, monographs and articles, answering the guiding problem that drives such research. Thus, these keywords were used: nurse, prostate cancer, prostate adenocarcinoma, man health, so combined to collect the studies included in the research. The language used was only the Portuguese and the year of publication of the articles included was limited to the years 1990-2014. Thereby, 217 articles were found in the databases, but after reading them, only 13 were used for the compilation of data of this literature review. Thus, as a result, it was observed that, currently, the nurse is the most able professional to practice care, guidance and assistance to prevent men about the problem of the prostate cancer. Moreover, as the study analysis, the nursing professional is usually responsible for the training of the team and can help demystify the existing preconceptions about the digital rectal examination in men. This way, the study noted that the nurse is an essential professional in the care and prevention of prostate cancer as it has specific knowledge that can assist in the transfer of information and guidelines relevant about this pathology.

Key-words: nurse, prostate cancer, Prostate adenocarcinoma, men healthcare.

\section{Resumen}

\section{Actuación del profesional de enfermería del trabajo en la prevención del cáncer de próstata}

El cáncer de próstata es una de las patologías más frecuentes y comunes en la población masculina, y la discusión de la prevención sigue siendo muy desfasada y necesita más atención en el marco de la salud pública. Pensando en ello, el objetivo de este estudio es evaluar cómo es el cuidado del profesional de enfermería del trabajo en la prevención del cáncer de próstata. Con este fin, este estudio incluye una revisión de la literatura, observando, a partir del uso de libros, tesis, monografías y artículos, responder al problema rector que guía esta investigación. Por lo tanto, se utilizaron las palabras clave: enfermera, cáncer de próstata, adenocarcinoma próstata, salud del hombre, en combinación, para recoger los estudios incluidos en la investigación. La lengua utilizada ha sido solo el portugués y el año de publicación de los artículos incluidos se limitó a los años 1990-2014. Por lo tanto, 217 artículos fueron encontrados en las bases de datos, pero después de leer en su totalidad, solo 13 fueron utilizados para la elaboración de los datos de esta revisión de la literatura. Así que como resultado se observó que, en la actualidad, el enfermero es el profesional más apto para practicar la atención, orientación y auxilio para prevenir el hombre con problemas de cáncer de próstata. Además, conforme análisis del estudio, el profesional de enfermería es generalmente responsable de la capacitación del equipo, y puede ayudar a desmitificar preconceptos existentes acerca del tacto rectal en los hombres. Por lo tanto, el estudio destaca que el enfermero es un profesional fundamental para el cuidado y la prevención del cáncer de próstata, ya que tiene conocimientos específicos que pueden ayudar en la transferencia de información y directrices pertinentes a esta patología.

Palabras-clave: enfermero, cáncer de próstata, enfermería del trabajo, salud del hombre.

\section{Introdução}

Como é de conhecimento, o câncer é "a doença da célula”, a qual causa desvio significativo em seu comportamento normal. O câncer de próstata por ser um problema frequente e passível de ocorrência, agrega maior atenção dos homens com idade acima dos 50. Por este fato, o presente estudo apresenta sua questão norteadora como sendo: "Como se dá a atuação do profissional de enfermagem na prevenção do câncer de próstata?”.
Assim, pensando nesse pressuposto, o objetivo geral da presente pesquisa é analisar a atuação do enfermeiro do trabalho no cuidado e prevenção do câncer de próstata. Dessa maneira, tem-se como objetivos específicos, explanar sobre a atuação do enfermeiro nesse cuidado, assim como as práticas preventivas e de promoção que podem ser utilizadas por este profissional com a finalidade de melhor combater tal problema.

Conforme salientam os autores Cezana et al. [1], o processo do cuidado/cuidar encontra-se 
vinculado às origens do ser humano, que por sua vez torna-se elemento essencial para a observância, realização e implementação de açóes que podem auxiliar na promoção, prevenção e recuperação do indivíduo.

Entretanto, como analisa Fernandes [2], o cuidado somente surge quando há existência de demanda específica para cuidado, bem como pode ocorrer quando alguém se importa com outra pessoa (nesse caso, o cuidador), ao observar possíveis demandas de cuidado. Por este fato, o cuidado e prevenção do câncer de próstata tem se tornado cada vez mais frequente, uma vez que o índice de homens acometidos por tal patologia é significativo.

Segundo a Sociedade Brasileira de Urologia [3], o adenocarcinoma prostático é a patologia que representa a segunda maior causa de morte na população masculina brasileira, ficando atrás apenas de doenças cardiovasculares e causas externas.

Logo, conceituando o câncer de próstata, pode-se dizer que, segundo Nogueira e Neves [4], esta patologia é uma doença causada pelo crescimento anormal e descontrolado das células da próstata, a qual acomete, principalmente, homens acima dos 50 anos.

Pensando nesse pressuposto, vê-se que açóes de educação em saúde, para o cuidado e prevenção do câncer de próstata, podem ser utilizadas com a finalidade de reduzir agravos advindos dessa patologia. Além disso, não só apenas as ocorrências, mas também o próprio número de mortes pode ser diminuído com o processo de prevenção dessa patologia. Por este fato, açóes educativas em saúde devem ser efetivadas, objetivando esclarecer o público masculino quanto aos fatores de risco e prevenção do câncer de próstata. É nesse cenário que o enfermeiro entra como orientador e educador em saúde.

Assim, com a finalidade de se conhecer as ações realizadas pelo profissional de enfermagem no cuidado e prevenção do câncer de próstata o presente estudo busca responder a questáo norteadora a partir de uma revisão de literatura.

Dessa forma, por acreditar-se que o enfermeiro é o profissional mais apto ao cuidado de educação em saúde para a prevenção do câncer de próstata, uma vez que seu conhecimento abrange ações específicas como orientaçôes aos pacientes, açóes informativas em grupos operativos, estímulo ao autocuidado e, sobretudo, orienta a família do paciente quanto a possíveis agravos, faz-se necessária a abordagem de açóes específicas utilizadas pelo profissional de enfermagem no enfrentamento e prevenção de tal patologia.

\section{Material e métodos}

Inicialmente foi feita a definição das palavras-chave a partir da consulta aos Descritores em Ciências e Saúde (DeCS). A busca dos artigos foi realizada nas bases de dados eletrônicas Medline, Literatura Latino Americana e do Caribe em Ciências da Saúde (Lilacs), Bireme, Scientific Eletronic Library Online (Scielo) e BDENF. Além disso, foram utilizados alguns livros com ano igual ou superior ao ano de 2005.

As palavras-chave utilizadas nas buscas foram: "enfermeiro", "câncer de próstata", "adenocarcinoma prostático" e "saúde do homem" de maneira combinada. A busca foi limitada ao idioma português, abrangendo apenas estudos que investigavam empiricamente o problema em questão. $\mathrm{O}$ ano de publicaçáo foi limitado aos anos de 1990 a 2014.

Foram encontrados 217 artigos nas bases de dados pesquisadas e, destes, 24 foram condizentes à proposta do presente estudo a partir da leitura dos títulos e resumos. Após leitura na íntegra, apenas 13 artigos foram aproveitados no estudo.

\section{Resultados e discussão}

As principais literaturas existentes auxiliaram na elaboração de três categorias temáticas, as quais tratavam em responder o problema de pesquisa proposto. As categorias geradas foram: "Conceitos e paradigmas envoltos ao câncer de próstata”; "A masculinidade e a prevenção do câncer de próstata"; "Práticas assistenciais em saúde realizadas pelo profissional de enfermagem".

\section{Conceitos e paradigmas envoltos ao câncer de próstata}

O câncer de próstata é a "doença da célula", a qual causa desvio do seu comportamento normal. Trata-se de uma doença de evolução lenta, cuja história natural é pouco conhecida e que, em sua escala, atinge mais homens com idade acima dos 50 anos [5]. É um dos principais problemas de saúde que acometem os homens na atualidade, entretanto existe a possibilidade de detecção precoce da doença a partir da efetivação de açóes simples, o que melhora significativamente o prognóstico [6]. 
Paiva [7] salienta que ainda existe muita vergonha por parte dos homens para a detecçáo de tal doença. Ainda existe muito preconceito envolto ao exame de toque retal, o que dificulta a detecção precoce do câncer de próstata. Nesse contexto, torna-se essencial o estabelecimento de estratégias assistenciais e de atividades educativas direcionadas para o público masculino. É nesse cenário que o enfermeiro pode atuar no combate e prevençáo do câncer de próstata.

Segundo o Ministério da Saúde o rastreamento de tal doença é possível a partir do exame de toque retal, o qual verifica o estado total da próstata do indivíduo; e o exame de dosagem de Antígeno Específico Prostático (PSA), que, por sua vez, identifica os níveis de glicoproteína originária da próstata na corrente sanguínea. O PSA, conforme afirmam os autores Amorim et al. [5], é um marcador biológico significativo na identificação do câncer de próstata. Logo, entende-se por detecção precoce o rastreamento de homens a partir de aspectos assintomáticos.

Dessa maneira, vê-se a prevenção como a melhor alternativa no combate ao câncer de próstata, embora pareça que, mesmo diante do diagnóstico precoce sejamos incapazes de fazer algo que modifique a predisposição genética dos homens.

Paiva [7:18] define prevençáo como:

\begin{abstract}
"A agregação de todas as atividades designadas para melhorar a saúde pessoal e pública por meio de estratégias, incluindo a implementação de modificaçóes comportamentais, educação em saúde, medidas de saúde. Portanto a prevenção da saúde visa melhorar as condiçóes de saúde do indivíduo e evitar o aparecimento de doenças. A prevenção específica tem como finalidade a proteção do indivíduo contra determinados agravos à saúde.”
\end{abstract}

Pensando nesse pressuposto, vê-se que o papel do indivíduo acaba sendo definido, assim como o controle do câncer de próstata pode ser efetivado a partir do conhecimento dos sintomas iniciais. É nesse contexto que o enfermeiro entra como orientador, auxiliando no processo de prevenção e detecção inicial de tal agravo.

\section{A masculinidade e a prevençáo do câncer de próstata}

No combate ao câncer de próstata, vê-se que os aspectos subjetivos dos indivíduos normalmente não são considerados, expondo-os a problemas relacionados à masculinidade envolta a tal doença. $\mathrm{O}$ ato de realização do toque retal é um procedimento delicado, uma vez que nesse processo a visão da masculinidade torna-se ameaçada [7].

De acordo com Cavalcante [8:41], "a cultura é extremamente marcante e, tanto quanto a vida biológica, interfere na formação da personalidade do ser humano". Logo, para que possamos compreender o homem em toda sua singularidade faz-se necessário que seus aspectos mais individuais sejam considerados, tais como sexo, idade, cultura, tempo, país que vive, estudando e atendendo-o como um ser sociocultural.

$$
\text { Logo, Paiva [7:24] analisa que: }
$$

"Em relação ao câncer de próstata, por se
tratar de região genital, de uma parte do
corpo considerada "reservada", permeada
de preconceitos e tabus, transmitidos de
geração em geração, o homem, ainda hoje,
século XXI, expressa e verbaliza a vergonha
na hora de se referir ao exame de toque retal."

Como o exame retal digital expóe o homem ao toque genital, o mesmo se vê remetido à questáo da homossexualidade, o que, de maneira machista, por muito interfere a continuidade dos atendimentos preventivos, assim como do cuidado à saúde do homem. Por este fato, se faz necessário o conhecimento de estratégias e conhecimento de novos conceitos que possam subsidiar um conhecimento mais específico, retirando paradigmas e pré-conceitos já estabelecidos pelos homens [5,9].

Segundo Gomes [10], como o toque envolve penetração, o mesmo também pode estar associado à dor física do indivíduo, bem como ao processo de violaçáo do corpo masculino. Outro receio envolto ao processo do toque é o medo da ereção durante o toque, que, em muitos casos, pode ser visto como prazer, pois no imaginário masculino, a ereçáo pode estar associada ao prazer, não conseguindo imaginá-la como fisiológica nesse caso.

Nascimento [11] salienta também que tal procedimento pode envolver um processo de grande constrangimento ao indivíduo, violando os principais preceitos masculinos. Percebe-se que ainda existem muitos mitos em relaçáo às questóes subjetivas envolvidas ao toque retal e, para desmistificá-las, demandará tempo para que novas pesquisas enfoquem o assunto. 
Diante do exposto, percebe-se que apesar da evoluçáo o homem tem em sua raiz preceitos antigos que precisam ser trabalhados de forma a desmistificar estas questóes visando melhorar a sua qualidade de vida.

\section{Práticas assistenciais em saúde realizadas pelo profissional de enfermagem}

Existem práticas assistenciais que são realizadas com o intuito de suprir as necessidades dos indivíduos. As práticas preventivas, realizadas principalmente pela equipe de Atenção Básica, fornecem informaçôes precisas e promoção de saúde para os indivíduos acometidos por determinadas doenças [7].

Paiva [7:18-19] salienta que:

"Sabidamente o rastreamento na população geral de doenças de baixa prevalência resulta em grande número de falsos positivos e, no caso do câncer de próstata, o verdadeiro diagnóstico será conseguido por técnicas invasivas. A este respeito, o INCA divulgou que, com exceção de dois ensaios clínicos em andamento atualmente na Europa (European Randomized Screening for Prostate Câncer Trial - ERSPC) e nos Estados Unidos (Prostate, Lung, Colorrectal and Ovarian Câncer Screening Trial - PCLO), os estudos disponíveis na literatura para avaliação da efetividade do rastreamento do câncer de próstata apresentam problemas metodológicos em seu desenho. $\mathrm{O}$ baixo valor preditivo positivo dos testes de rastreamento indica uma elevada proporção de resultados falso-positivos, e, conseqüentemente, um alto índice de realização de biópsias desnecessária."

Por este fato, o rastreamento de homens assintomáticos deve ser efetivado de maneira diferenciada, associando as características a danos importantes.

Com base nesses preceitos, o profissional de enfermagem deve oportunizar estratégias de educação em saúde para o cuidado e processo de prevenção do câncer de próstata, tais como:

- Oportunizar experiências educativas com grupos de homens nos serviços de saúde e centros comunitários de modo a favorecer a divulgação de conhecimentos, esclarecendo dúvidas sobre o câncer de próstata e outros assuntos correlacionados à saúde masculina;
- Promover programas de educaçáo continuada para os profissionais das diferentes categorias nos serviços, ampliando o conhecimento dos mesmos para os objetivos de um programa de detecção precoce para o câncer de próstata;

- Sensibilizar a equipe de saúde a fim de realizar o rastreamento do homem em idade acima dos 50 anos com o intuito de possibilitar orientaçóes e a possível prevenção do câncer de próstata neste indivíduo;

- Capacitar e motivar os profissionais que atuam na educação em saúde a fim de instrumentalizá-los para orientação dos indivíduos e esclarecimento de dúvidas;

- Priorizar a orientação a homens que apresentem barreiras de acesso, baixa escolaridade e dificuldades em solicitar o exame;

- Promover maior interface do trabalho das equipes de saúde da família, de modo a sensibilizar e oportunizar homens para rastreamento;

- Propiciar a divulgaçáo de programas e de grupos operativos como veículo de educação em saúde;

- Realizar trabalho de sensibilização cotidiana dos profissionais de saúde para a motivação e implementação de outra lógica nos serviços, que contemple as atividades de prevenção e promoção da saúde, além de assistência à doença [5-7,12,13] .

Dessa forma, acredita-se que o profissional de enfermagem é o mais apto a orientar tanto o indivíduo quanto os profissionais de saúde acerca do cuidado e prevenção do câncer de próstata, subsidiando a melhora do atendimento, qualidade e humanização no mesmo para os potenciais portadores de tal patologia.

\section{Conclusão}

Após análise dos resultados, percebe-se claramente a importância do atendimento efetivado pelo enfermeiro em nível de Atenção Primária, o qual se encontra envolto, principalmente, à humanização do atendimento, à consideraçáo da individualidade do cliente, à forma de orientar o cliente e sua família em saúde, preconizando a prevenção do câncer de próstata a fim de se evitar futuros agravos ao paciente. Além disso, o profissional de enfermagem consegue propiciar a capacitação de outros profissionais, orientando-os acerca dos cuidados e prevenção de tal doença.

Há de se dizer ainda que as orientações realizadas em grupos operativos, saneando dúvidas e dando 
orientaçóes pertinentes ao cuidado e prevenção em saúde, tornam-se de grande valia para o indivíduo acima dos 50 anos, uma vez que este se encontra mais ativo na faixa de risco do câncer de próstata.

$\mathrm{O}$ enfermeiro, nesse contexto, atua como profissional chave no cuidado em saúde, tornando-se indispensável na orientaçáo e cuidado para a prevenção do câncer de próstata.

Portanto, para o real desenvolvimento do cuidado em saúde, bem como para o sucesso do tratamento do possível paciente com câncer de próstata, faz-se necessário o envolvimento e a polivalência de toda equipe de saúde, pois a família e/ou indivíduo possuem diagnósticos diferenciados, apresentando diferentes demandas de tratamento, logo, sua individualidade/subjetividade deve ser considerada.

Assim, é de fundamental importância que os profissionais possuam conhecimentos diferenciados e adequados, relativos ao problema apresentado, de maneira que todos profissionais possam se adequar, planejar e executar açóes específicas para o cuidado e prevenção em saúde de homens com potencial desenvolvimento do câncer de próstata.

\section{Referências}

1. Cezana AR, Boone MBF, Olympio PCAP, Amorim MHC, Oliveira MJM. Perfil dos cuidadores familiares de pacientes acamados assistidos por um serviço de assistência domiciliar. Rev Bras Pesq Saúde 2011;13(2):43-8.

2. Fernandes JM. O papel do cuidador frente ao paciente acamado e a responsabilização da equipe de saúde da fa- mília. Araçuaí: Faculdade de Medicina (UFMG); 2010.

3. SBU. Sociedade Brasileira de Urologia. Alerta para prevençáo do câncer de próstata. Rio de Janeiro: Sociedade Brasileira de Urologia; 2008. p.5-13.

4. Nogueira HL, Neves JB. Prevençáo do câncer de próstata: atuação dos enfermeiros nas unidades de atenção primária a saúde. Revista Enfermagem Integrada 2013;6(1):1098-1109.

5. Amorim VMSL, Barros MBA, César CLG, Goldbaum M, Carandina L, Alves MCGP. Fatores associados à realizaçáo dos exames de rastreamento para o câncer de próstata: um estudo de base populacional. Cad Saúde Pública 2011;27(2):347-56.

6. Paiva EPP, Motta MCS, Griep RH. Conhecimentos, atitudes e práticas acerca da detecção do câncer de próstata. Acta Paul Enferm 2010;23(1):88-93.

7. Paiva EP. Conhecimentos, atitudes e práticas acerca da detecçáo do câncer de próstata. Rio de Janeiro: UFRJ; 2008.

8. Cavalcante C. Sexualidade do homem na terceira idade. Revista Brasileira de Sexualidade 1990;15(2):39-49.

9. Lara M. Com todas as letras - O estigma do câncer por quem enfrentou esse inimigo silencioso e cruel. Rio de Janeiro: Record; 2005.

10. Gomes R. Sexualidade masculina e saúde do homem: proposta para uma discussão. Revista Ciências e Saúde Coletiva 2003;8(3):825-29.

11. Nascimento MR. Câncer de próstata e masculinidade: motivaçôes e barreiras para a realização do diagnóstico da doença. Caxambu: Abesp; 2005.

12. Souza LM, Silva MP, Pinheiro IS. Um toque na masculinidade: a prevenção do câncer de próstata em gaúchos tradicionalistas. Rev Gaúch Enferm 2011;32(1):151-8.

13. Medeiros AP, Menezes MFB, Napoleáo F. Fatores de risco e medidas de prevençáo do câncer de próstata: subsídios para a enfermagem. Rev Bras Enferm 2011;64(2):385-8. 